\title{
From endometriosis to pregnancy: which is the "road-map"?
}

\author{
Errico Zupi ${ }^{1}$, Giovanna De Felice ${ }^{2}$, Francesca Conway ${ }^{1}$, Francesco Martire ${ }^{1}$, Caterina Exacoustos ${ }^{1}$, Gabriele Centini $^{2}$, \\ Lodovico Patrizi ${ }^{1}$, Emilio Piccione ${ }^{1}$, Lucia Lazzeri ${ }^{2}$ \\ ${ }^{1}$ Department of Biomedicine and Prevention, Obstetrics and Gynecological Clinic, University of Rome "Tor Vergata," Rome - Italy \\ ${ }^{2}$ Department of Molecular and Developmental Medicine, Obstetrics and Gynecological Clinic, University of Siena, Siena - Italy
}

\begin{abstract}
In the last decade, pregnancy was considered as a therapeutic period for patients affected by endometriosis and painful symptoms. However, several studies have taken into consideration how endometriosis affects pregnancy achievement and pregnancy development, including obstetric complications. The adverse effects of endometriosis on the development of pregnancy include miscarriage, hypertensive disorders and pre-eclampsia, placenta previa, obstetric hemorrhages, preterm birth, small for gestational age, and adverse neonatal outcomes. The aim of this review is to analyze the current literature regarding the relationship between different forms of endometriosis (endometrioma, peritoneal endometriosis, deep endometriosis) and infertility, and the impact of endometriosis on pregnancy outcomes.
\end{abstract}

Keywords: Endometriosis, Infertility, Obstetric complications, Pregnancy

\section{Introduction}

Endometriosis is a benign chronic gynecological disease that affects up to $50 \%$ of infertile women. The mechanisms by which endometriosis affects infertility are different and not fully understood. They range from distortion of pelvic anatomy to immunological disturbances and endocrine abnormalities (1). It also has been suggested that chronic inflammatory response - a typical trait of this disorder - plays an important role in reducing fertility (2). Adenomyosis, which is frequently associated with endometriosis, affects fertility as well (3). Endometriosis can affect various organs such as the ovaries, the posterior Douglas cul-de-sac, the bowel, the bladder, and the ureters. The severity of organ involvement and the stage of the disease are correlated with reduced fertility (4). Harb et al (2), in a recent meta-analysis, revealed a significant reduction of implantation rate and pregnancy rate in patients with severe endometriosis (stage III/IV for the American Society of Reproductive Medicine classification) undergoing in vitro fertilization (IVF) treatment. Furthermore, the presence of an endometrioma in women

Accepted: October 3, 2017

Published online: November 1, 2017

\section{Corresponding author:}

Errico Zupi

Department of Biomedicine and Prevention

Obstetrics and Gynecological Clinic

University of Rome "Tor Vergata,"

Via Montpellier

00100 Rome, Italy

ezupi@libero.it with severe endometriosis is associated with significantly lower pregnancy rates when compared with women without endometriomas (5). The negative impact of surgical excision of an endometrioma on ovarian reserve and on anti-Müllerian hormone (AMH) levels has been demonstrated (6). Despite the fact that surgery for deep infiltrating endometriosis (DIE) may increase spontaneous conception (7), recent guidelines do not recommend surgery with the single aim of improving assisted reproductive technology (ART) outcomes (6).

Several studies have taken into consideration how endometriosis affects pregnancy achievement and pregnancy development, including obstetric complications (8). Pregnancy complications accompanying preexisting endometriosis may be explained by some pathogenic mechanisms, such as endometriosis-related chronic inflammation (9), the presence of adhesions and their mechanical implications (10), and the invasion of decidualized ectopic endometrium in to the vessels wall $(11,12)$.

The aim of this review is to analyze the current literature regarding the relationship between different forms of endometriosis (endometrioma, peritoneal endometriosis, DIE) and infertility, and the impact of endometriosis on pregnancy outcomes.

\section{Does endometrioma mean infertility?}

Endometrioma is a frequently encountered entity in ovarian surgery and affects from $17 \%$ to $44 \%$ of patients with endometriosis (13). The most common symptoms of ovarian endometriomas include dysmenorrhea, chronic pelvic pain, dyspareunia, and infertility. Transvaginal ultrasound (TVU) has a high diagnostic accuracy for the diagnosis of endometrioma 
and the prediction of the presence of DIE and related pelvic adhesions (14).

The mechanisms of endometriosis-related infertility remain largely unknown $(15,16)$. Several causes have been previously implicated, from anatomic distortion and tubal occlusion due to pelvic adhesions to less well-described factors, such as inflammatory cytokine-mediated impairment of endometrial receptivity and oocyte quality (17).

The impact of ovarian endometriomas on spontaneous conception and on ART outcomes is controversial. Several studies have shown how the presence of endometriomas can affect the amount and quality of the adjacent ovarian follicles (18), and reduce the rate of spontaneous ovulation (19). It has been highly demonstrated that women with endometriomas show lower baseline AMH levels (20). Several studies have shown that $\mathrm{AMH}$ levels appear to be reduced 1 month after ovarian surgery; however, they tend to recover to baseline values after 6 months (21-25). Furthermore, considering long-term followup, some authors stated that AMH levels temporarily decreased after laparoscopic cystectomy with complete recovery of preoperative $\mathrm{AMH}$ values after 12 months of surgery $(26,27)$.

In addition, the presence of endometriomas in women with severe endometriosis is associated with significantly lower pregnancy rates following IVF treatment when compared with women who suffer from severe endometriosis without endometriomas (5).

The management of an asymptomatic ovarian endometrioma in patients with infertility is controversial. The surgical approach remains a necessary part of the treatment algorithm (28). The 2008 European Society of Human Reproduction and Embryology (ESHRE) guidelines on endometriosis recommend laparoscopic ovarian cystectomy for patients with an endometrioma $\geq 4 \mathrm{~cm}$ in diameter, to improve the access to follicles and the ovarian response (29-31). Among the available surgical techniques, ovarian endometrioma excision has been shown superior to drainage and coagulation alone in preserving fertility and controlling pain, and is associated with a lower risk of recurrence (32). However, other studies have reported a reduction of the ovarian reserve of the patient following surgical excision of endometriomas (33, 34). Therefore, the ESHRE guidelines underline the importance of accurate presurgical counseling regarding this risk, with a detailed explanation of the potential negative effect of surgery on future fertility (29).

Although the close relationship between endometriosis and infertility has been studied for decades, we have yet to identify the factor that impacts negatively on fertility the most: could it be the mere presence of an endometrioma per se, or the effects of surgery on ovarian reserve? Histologic findings showed that ovarian follicles in the ovarian cortical layer of endometriosis women are decreased in number and are more atresic. A recent review showed that the local intrafollicular environment of patients with endometriosis is characterized by alterations to the granulosa cells compartment, resulting in a worsening of oocyte quality and ovarian physiology $(35,36)$.

Patients with fertility desires should receive detailed counseling focusing on the ratio between the risks and the benefits of both fertility options versus surgical intervention.

Rossi et al (37) in a recent meta-analysis showed that a history of surgery for endometriosis or endometriomas is associated with poor IVF outcomes. According to some authors, pregnancy rates are significantly improved when patients are treated with surgery followed by ART, even the subgroup of patients with "asymptomatic" endometriomas (38). According to other authors, there is no significant difference in terms of IVF/intracytoplasmic sperm injection (ICSI) outcomes, between the group of patients that received surgical treatment of endometrioma and the group who did not (39). Hamdan et al (39) demonstrated that patients with endometriomas had similar reproductive outcomes with IVF/ICSI compared to those without the disease, but they had a higher rate of cycle cancellation. According to these authors, the surgical treatment of endometrioma may help optimize the IVF/ICSI results, despite the potential detrimental impact (39). The main factors that can be involved in the prediction of surgical damage on ovarian reserve are age, bilateral ovarian cystectomy, and repeat endometrioma intervention $(40,41)$. The impact of surgery on the ovarian reserve is well known. For instance, Streuli et al (42) demonstrated that $\mathrm{AMH}$ levels were significantly decreased in patients with endometriosis who had prior endometrioma surgery, compared to women who did not undergo surgery. In another study, Somigliana et al (33) showed significantly impaired IVF outcomes in patients previously operated on for bilateral endometrioma. Chapron et al (43) stressed the concept that before thinking about surgical treatment, it is needful to consider the association between ovarian endometrioma and severe DIE, especially in patients that complain of painful symptoms. In fact, it has been demonstrated that ovarian endometriomas are significantly associated with other forms of the disease, such as pelvic deep infiltrating endometriosis, ovarian adhesions, and pouch of Douglas obliteration. Surgery for endometriosis can be very complex and, in some cases, when more than a simple endometrioma excision is required, the gynecologist may request an interdisciplinary approach to safely perform an extensive dissection within the pelvis. Incomplete removal of ovarian disease can lead to partial resolution of symptoms and the need for additional surgery or medical treatment (44). Shervin et al (45) evaluated the reproductive outcome following laparoscopic excision of endometriosis in infertile women with DIE, with and without endometrioma. The researchers found equal pregnancy rates in women who underwent surgery for DIE with endometrioma, compared with patients who underwent DIE excision in the absence of endometrioma (35.6\% vs. $39.5 \%$, respectively). This study shows how the removal of DIE plays a more important role on fertility than ovarian cystectomy.

Despite that endometriomas are significantly associated with infertility, they should not be surgically removed for the sole purpose of improving fertility/reproductive outcomes, as demonstrated by currently available research. Greater attention should be paid to the preoperative ovarian reserve status of the patient and to the possible ovarian response to stimulation, since these two factors alone are greater predictors of reproductive outcomes compared to the sole presence of an endometrioma (46).

\section{Peritoneal endometriosis and infertility}

Minimal or mild endometriosis (stages I/II) mainly comprise the forms characterized by peritoneal endometriotic implants 
and the presence of filmy adhesions between the various genital organs, particularly on the ovaries or the fallopian tubes. This form of endometriosis is frequently identified in infertile patients, especially in cases of unexplained infertility (47), even if its real impact on fertility is still under discussion. Indeed, while some authors agree that superficial peritoneal endometriosis is clearly associated with infertility $(48,49)$, according to others this relationship is questionable (50). The mechanisms through which peritoneal endometriosis reduces fertility are various and involve peritoneal fluid and cavity microenvironments (51), plus reduced mobility of the fallopian tubes and ovaries related to pelvic adhesions. These mechanisms could explain the advantages yielded by adhesiolysis and the complete removal of superficial peritoneal implants (52). Marcoux et al (49) have shown that laparoscopic resection or ablation of minimal and mild endometriosis enhances fecundity in infertile women while involving minimal risk. In these patients, intrauterine insemination (IUI) with controlled ovarian stimulation improves fecundity. Nonetheless, IVF remains the most efficient method, allowing pregnancy in case of tubal infertility (53). In agreement with these findings, Boujenah et al (54) have shown that ovarian stimulation after laparoscopic treatment for superficial peritoneal endometriosis-related infertility was associated with an increased pregnancy rate (54). Opøien et al (55) found similar results, demonstrating how the surgical eradication of minimal and mild endometriosis increased the birth rate both spontaneously and after IUI. In that study, 399 women underwent complete surgical removal of lesions, whereas 262 women underwent diagnostic laparoscopy only; significantly improved implantation rates (30.9\% vs. $23.9 \%$ ), pregnancy rates $(40.1 \%$ vs. $29.4 \%)$, and live-birth rates per ovum retrieval $(27.7 \%$ vs. $20.6 \%$ ) were seen in the full surgery group compared to the diagnostic laparoscopy group. Furthermore, surgical removal of minimal and mild endometriotic lesions gave a shorter time to first pregnancy and a higher cumulative pregnancy rate (55). These findings were in line with previous studies (56-59) and inconsistent with others (60, 61). In fact, other authors did not confirm the same result. In particular, in 1999, Parazzini (62) conducted a study on patients with minimal and mild endometriosis. The patients were randomly assigned to resection/ablation of visible endometriosis (54 patients) or diagnostic laparoscopy only (47 patients). After laparoscopy, all patients tried to conceive spontaneously. After the follow-up period, 12 (24\%) patients in the resection/ablation group and $13(29 \%)$ in the no treatment group conceived with no significant difference in the 2 groups. In conclusion, there is no treatment option in subfertile patients with minimal or mild endometriosis. The optimal management should be identified based on clinical presentation and patient need.

\section{Deep endometriosis: anterior endometriosis}

Urinary tract endometriosis (UTE) is a rare form of DIE affecting $0.3 \%-12 \%$ of all women with endometriosis (63) and $14 \%-20 \%$ of all DIE patients (64). Of the various forms of UTE, the bladder is the most common localization of endometriosis $(85 \%)$, followed by the ureter $(9 \%)$, the kidney $(4 \%)$, and the urethra (2\%) (65). The symptomatology of this disorder is not characteristic and includes nonspecific abdominal and urinary symptoms (66), such as urinary frequency, urgency, dysuria (70\% of patients with bladder endometriosis), and hematuria (20\%-35\%). Ureteral involvement may be asymptomatic, sometimes leading to the silent loss of renal function. Infertility is also common in women with urinary endometriosis. For patients suffering from severe endometriosis, the best treatment is the complete removal of all endometriotic lesions involving the bladder, the ureter, and the bowel, especially when the symptomatology is resistant to medical therapy. Surgery is the first option in cases of UTE-related hydronephrosis. In fact, after the surgical treatment of these lesions, most patients report satisfaction with good pain relief. In addition, it has been suggested that the recurrence rate of bladder endometriosis is lower than that of other forms of endometriosis (67). Attention should be paid to the possible association of anterior endometriosis with other forms of the disease; in fact, it has been demonstrated that anterior endometriosis is rarely isolated and the risk of ureteral endometriosis increases if a rectovaginal nodule is larger than $3 \mathrm{~cm}$. Therefore, in planning surgery for DIE, it is necessary to perform other diagnostic tests, such as urinary tract ultrasonography, urography, or magnetic resonance imaging (MRI) $(64,68,69)$. Saavalainen et al (70) also found an association between UTE lesions and other DIE localizations, highlighting the need for detailed preoperative imaging, adequate surgical planning, and a multidisciplinary team of specialists, in order to reduce the rate of postoperative complications. If the role of surgery as treatment for painful and urinary symptoms is clear, on the other hand, the role of the surgical approach to treat infertility is not so defined. Indeed, according to the ESHRE guidelines, in subfertile patients with DIE, there is no consensus concerning the first-line management of infertility (surgery vs. ART) and there is not enough evidence to propose surgery before ART (6). Soriano et al (71) have studied the reproductive outcome in patients with bladder endometriosis after laparoscopic surgery. The study included 69 patients that were surgically treated for bladder endometriosis. Of these, 21 patients had full-thickness bladder penetration and underwent partial cystectomy while the remaining 48 patients had partial bladder penetration and underwent partial-thickness excision of the detrusor muscle. Only 42 patients of the 69 included in the study desired to conceive. Of these 42 patients, 36 (85.7\%) conceived after surgery; 16 (47\%) conceived spontaneously, and 18 (53\%) conceived after IVF treatment. The average duration of infertility was 22.5 months. Comparing those who underwent full resection with those who underwent partial resection of bladder endometriosis, no difference in the overall birth rate was observed. Saavalainen et al (70) also have studied the assessment of fertility and the incidence of pregnancy and delivery complications following surgical treatment of UTE. They found that women affected by UTE conceived better than what was expected from the results of previous studies concerning DIE (72-74), but needed infertility treatment.

Currently, laparoscopic management could be considered the treatment of choice for patients with bladder endometriosis. Several studies have shown that laparoscopic surgery has long-term benefits in terms of quality of life, and it frequently leads to the remission of clinical symptoms (75). It has been demonstrated that medical options are more often purely palliative and do not determine the recovery from 
the disease (76). Furthermore, the majority of medical treatments are incompatible with a desire to conceive.

In conclusion, for symptomatic patients with bladder endometriosis who wish to conceive or desire to do so in the future, laparoscopic surgery is the most favorable approach and the first treatment to consider.

\section{Deep endometriosis: posterior endometriosis}

It is estimated that more than $20 \%$ of women with endometriosis are affected by posterior endometriosis. Posterior endometriosis is defined by ESHRE as the infiltration of endometriotic tissue by more than $5 \mathrm{~mm}$ beneath the peritoneum (6). The most severe forms of posterior DIE are considered to be the colorectal lesions, which involve the rectosigmoid junction and rectum. These endometriotic lesions are often associated with others, which could involve the uterosacral ligaments, the torus uterinus, the parametrium, the vagina, and the ovaries (77). While it has been largely demonstrated that the complete surgical excision of DIE lesions leads to an improvement of quality of life with persistent relief from symptomatology (78-85), the impact of colorectal endometriosis alone on fertility is still unclear. Adamson and Pasta (86), in their meta-analysis, have demonstrated that a complete surgical removal of lesions in patients with severe DIE leads to an increase of the spontaneous pregnancy rate with a differential gain in pregnancy of $39 \%$. In 2009 , Stepniewska et al (87) compared 3 groups of infertile patients: the first group consisted of 60 patients who underwent surgery for DIE with colorectal segmental resection; the second group was 40 patients with bowel involvement who underwent endometriosis removal without bowel resection; and the last group included 55 women who underwent surgery for DIE and at least 1 endometrioma but without bowel involvement. After a mean follow-up period of 2 years, 17 patients (35\%) conceived after surgery in the first group; 8 patients $(21 \%)$ in the second group became pregnant, and 32 patients $(70 \%)$ in the last group conceived. There was a significant difference between the 3 groups both with regard to the total pregnancy rate and in terms of the time it took to conceive. The presence of intestinal endometriosis was associated with the lower pregnancy rates. According to these results, the presence of bowel endometriosis negatively influences the fecundity, and the postoperative fertility is improved when segmental bowel resection is performed (87). Darai et al (88) have recently published a review article on the potential benefits of ART and surgery on fertility outcomes. Although this paper confirms the potential benefit of surgery on fertility for patients with colorectal endometriosis, it is unclear whether this treatment should be the first line or limited to cases of ART failure. In fact, spontaneous pregnancy rates (PR) in patients undergoing DIE resection without the removal of colorectal lesions was $26.5 \%$ (95\% confidence interval [CI], 14-39); PR after medically assisted reproduction was $27.4 \%(95 \% \mathrm{Cl}$, 19-35) and the overall PR was $37.9 \%(95 \% \mathrm{Cl}, 29-37)$. After colorectal surgery, among the 855 patients with and without proved infertility, the spontaneous PR was $31.4 \%(95 \% \mathrm{Cl}$, 28 34) without difference between the groups. The overall PR after colorectal surgery was $51.1 \%(95 \% \mathrm{Cl}, 48-54)$. According to other authors, both spontaneous pregnancy rates and ART pregnancy rates improved after a complete surgical removal of DIE lesions. Centini et al (89) evaluated fertility outcomes after laparoscopic excision of deep endometriosis and correlated these findings with lesion number, size, and location (anterior, posterolateral, pouch of Douglas, and multiple locations). In this retrospective study, they found a higher pregnancy rate by both spontaneous conception and ART after surgical excision of multiple lesions. After a mean follow-up of 22 months, the overall pregnancy rate was $54.78 \%$ with a live-birth rate of $42.6 \%$. In the group of patients who tried to conceive spontaneously, the overall pregnancy rate was $60 \%$ : $38.5 \%$ spontaneously and $21.4 \%$ by ART. Moreover, according to the results of this study, the surgical management of isolated DIE lesions influenced the fertility irrespective of their location and size, with no differences in pregnancy rates when comparing isolated lesion size and disease location.

\section{Endometriosis-related complications during pregnancy}

Usually, the typical hormonal milieu of pregnancy favors the regression of endometriotic lesions. Unfortunately, some critical conditions linked to endometriosis may occur in pregnancy. Despite the fact that the evidence is scarce, clinicians should be aware of these adverse events and suspect rare but life-threating conditions for both the mother and the fetus when an abdominal pain occurs in a pregnant woman.

\section{Spontaneous hemoperitoneum in pregnancy}

The prevalence of endometriosis-related spontaneous hemoperitoneum is estimated to be about $0.4 \%(90)$. Brosens et al (91) reviewed studies on spontaneous hemoperitoneum in pregnancy (SHiP) published during the last 20 years and assessed an incremented risk in nulliparous women $(70 \%$ of cases) and in pregnancies conceived through IVF. They did not find differences in age, parity, or gestation in women with and without endometriosis, but all cases of spontaneous hemoperitoneum after IVF occurred in women with endometriosis (91). There are many hypotheses regarding the pathogenesis of endometriosis related SHiP. The invasiveness of endometriosis may be responsible for the development of SHiP, but no apparent correlation between spontaneous hemoperitoneum and stage of endometriosis was found (91). The infiltration of decidualized endometriotic tissue into the vessel wall may predispose to tissue rupture, causing increased back pressure, edema, angiogenesis, and vascular permeability (92). A possible alternative explanation is based on the involution of the decidualized endometrium. After reviewing 20 studies published over a 20-year period from 1987 to 2008, Viganò et al (90) identified endometriosis in 13 of the 25 women who experienced SHiP. The hemoperitoneum could originate from the dilated utero-ovarian vessels, the varicosities on the uterine surface, or the thin vessels located in the decidualized stroma of endometriosis localizations. The clinical presentation of SHiP was described as a sudden onset of abdominal pain associated with hypovolemic shock, a marked reduction of hemoglobin levels, and possible intrauterine fetal death in the absence of vaginal bleeding; hemothorax and extensive decidualization mimicking malignancy could be atypical clinical features. When an acute abdominal pain and hypovolemic shock 
occurs in a pregnant nulliparous woman, especially in the third trimester or in the postpartum period, particularly in the presence of a history of endometriosis, SHiP must be suspected. Nonetheless, advanced gestational age, maternal obesity, and the presence of blood clots can limit its potential. Brosens et al (91) underlined the failure of abdominal ultrasound examination in the studies included in their review; in most cases the diagnosis was only established at laparotomy, which was carried out for maternal reasons (hypovolemic shock and progressive anemia), for fetal reasons (fetal distress), or both. Explorative laparotomy was carried out in $90 \%$ of cases, while laparoscopy was performed in only 1 hemodynamically stable patient (93). Various critical situations must be taken into account in the differential diagnoses, including uterine rupture, abdominal pregnancy, placental abruptio, HELLP syndrome, and liver or spleen rupture (90). Surgery is the gold standard for the treatment of spontaneous hemoperitoneum. Since the posterior wall of the uterus and the parametrium are the most frequent bleeding sites, surgical access may be impaired by the pregnant uterus. A greater knowledge of spontaneous hemoperitoneum and the correlation with endometriosis is needed to improve maternal and neonatal outcomes.

\section{Bowel perforation}

Bowel endometriosis can be found in $5 \%-12 \%$ of women affected by endometriosis, and the sigmoid colon is the most frequently interested site, followed by the rectum, ileum, appendix, and cecum (94). Every layer of the bowel wall can be invaded by endometriosis, but the subserosa is the most common site. Bowel perforation is a complication of endometriosis, which can also occur during pregnancy. Adhesions, related to the disease itself or to prior surgery, might cause trauma during uterine growth, and extensive decidualization might weaken the bowel wall. In a review conducted by Viganò et al (90) the prevalence of spontaneous bowel perforations remained unknown. They took into account a recent review by Setúbal et al (95), which described 12 case reports of bowel perforation during pregnancy: 2 involved the small intestine, 1 the cecum, 3 the appendix, and 6 the rectosigmoid colon. Seven of the 12 perforations occurred between the 26 th and 37 th week of gestation, and the remaining 3 in the immediate postpartum period. According to Setúbal et al (95), the majority of bowel perforations were not promptly diagnosed during the exploratory laparotomy, requiring a repeated laparotomy. Treatment for bowel perforation during pregnancy is mostly represented by segmental intestinal resection, and sometimes a Hartmann procedure is needed.

\section{Uterine rupture}

Viganò et al (90) reviewed the literature on pregnancy outcomes in women with endometriosis and found 3 cases of endometriosis-related uterine ruptures $(90,96-98)$. A previous scar in the uterus represents a major risk factor during pregnancy. In these 3 case reports, the patients had undergone surgery for endometriosis (excision of a rectovaginal nodule, bilateral ovarian cystectomy, and excision of cervical endometriosis). Van De Putte et al (96) conducted an emergency cesarean delivery (CD) at 37 weeks of gestation due to signs of fetal distress during labor in a woman who had undergone the excision of a rectovaginal nodule 6 years before pregnancy. They detected a rupture on the posterior wall of the lower uterine segment (96). Chen et al (98) found a uterine lesion during manual exploration of the uterine cavity due to a retained placenta after vaginal delivery at term in a patient who had undergone excision of a rare cervical endometriotic cyst 1 year before pregnancy. In all cases, healthy babies were born and no maternal death was reported (98). Sholapurkar et al (99) performed a total abdominal hysterectomy in a woman with important vaginal bleeding, who had undergone $C D$ for an arrested labor progression 6 weeks previously. Endometriosis was found in the histological exam of the cesarean section scar; it involved the full thickness of isthmus from which the bleeding had started (99). A series by Zwart et al (100) described 2 women with a previous history of endometriosis who suffered an unscarred uterine rupture. Chester and Israfil-Bayli (101) published a case report on an emergency $C D$ for fetal distress performed on a nulliparous woman who had undergone a diathermy treatment of stage IV endometriosis located in the lateral wall of the pelvis and on the uterine surface. For this reason, authors speculate whether endometriosis should be considered as a risk factor for uterine rupture during delivery. The limit of this study is the impossibility to rule out the chance of previous undiagnosed perforation during the evacuation performed 2 years before.

\section{Endometriosis and pregnancy outcomes}

Recent studies have demonstrated the relevant impact of endometriosis not only in reducing fertility but also in affecting the pregnancy outcomes. Different mechanisms are thought to be involved: increased inflammation in the peritoneal cavity, molecular and functional abnormalities of the eutopic endometrium (102), poorer oocyte quality, progestogen resistance, defective remodeling of the myometrial spiral arteries, defective deep placentation, and decidualization of the endometriotic tissue $(103,104)$. The current evidence does not allow full understanding of the real connection between endometriosis and adverse pregnancy outcomes. No studies have focused on the histopathology of the placenta in women with endometriosis, searching for alterations that may explain the impaired decidualization and placentation. However, a wide spectrum of major obstetrical complications frequently affect women with endometriosis.

\section{Miscarriage}

Many studies have tried to find a relationship between endometriosis and miscarriage, and a positive link between treatment and the reduction of abortion rates. Most of these studies were retrospective, enrolling women with endometriosis from a group of infertile women, without controls and with insufficient follow-up (105-107).

A population-based cohort study conducted by Saraswat et al (108), using the national Scottish-linked data extended over a period of 30 years from 1981 to 2010, compared all women in Scotland diagnosed with endometriosis for the first time during surgery (laparoscopy or laparotomy) between 
1981 and 2009, who went on to achieve pregnancy, with a random sample of patients with no previous diagnosis of endometriosis, who had a pregnancy during the same time period. Women with endometriosis had a significantly higher likelihood of early pregnancy complications - in particular miscarriage and ectopic pregnancy (108).

Seven retrospective studies investigated the link between miscarriage and endometriosis regardless of the treatment (109-116) and only 2 of them observed an association. Vercellini et al (113) recruited nulligravid women with natural conception and subdivided them into 4 groups based on the type of endometriosis: rectovaginal, ovarian and peritoneal, only ovarian, and only peritoneal. At a crude analysis, the frequency of miscarriage was significantly higher in women with ovarian endometriomas and peritoneal lesions, but the difference was no longer significant at age-adjusted analysis. Comparing the outcome of both spontaneous and ART pregnancies in women with endometriosis versus a nonaffected population, Aris (114) found an increased rate of spontaneous abortion in women with endometriosis versus controls. Supporting the idea that endometriosis may represent a permanent problem throughout the whole pregnancy, in this retrospective cohort study, the author also reported a significant association between endometriosis and stillbirth. The other 4 retrospective studies did not find any association, but we can underline that in the studies finding an association, pregnancies by ART techniques were not excluded. Opposite conclusions were drawn in the retrospective nonrandomized trial conducted by Matorras et al (116), which compared infertile women with a histological diagnosis of endometriosis and infertile women without endometriosis showing a similar abortion rate in both groups (116). In contrast, in the retrospective nonrandomized trial conducted by Hjordt Hansen et al (117) comparing women with endometriosis to a population of women without endometriosis, a higher rate of miscarriage was found in the case group throughout the whole study period, which is the longest follow-up conducted so far.

A systematic review and meta-analysis by Barbosa et al (118) compared the ART outcome in women with and without endometriosis and at different stages of disease; they observed a higher risk of miscarriage in women with endometriosis, although it did not seem to vary with different stages of the disease.

In a case control study, Leonardi et al (119) evaluated whether women with endometriosis achieving singleton pregnancies with IVF showed an increased risk of miscarriage. Data revealed that the risk of miscarriage is not increased in women with endometriosis achieving pregnancy with the use of IVF. Some studies have focused on trying to find a positive link between pregnancy outcomes and endometriosis treatment. In the retrospective study of Pittaway et al (120), the abortion rate was significantly higher in the endometriosis group than in the fertile nonendometriosis group, and when they examined the pregnancy outcomes after treatment, there was a significant reduction in the frequency of spontaneous miscarriage in the endometriosis group. Two prospective, randomized, controlled trials studied the effect of the laparoscopic surgical treatment on pregnancy outcomes, including miscarriage, in patients with minimal to mild endometriosis. Neither study was able to show a significant reduction in miscarriage rates after laparoscopic treatment $(121,122)$. According to these results, we cannot draw any conclusion regarding the association between endometriosis and miscarriage.

\section{Preterm birth}

Preterm birth is a cause of neonatal morbidity and future adult disease (123). The increased inflammation status represents an important link between endometriosis and preterm birth; the consequent altered balance of the endometrial immunendocrine pathway may influence the trophoblast implantation and cause preterm birth (102). Conti et al (124) evaluated pregnancy, delivery, and neonatal outcomes in singleton primiparous versus multiparous women with and without endometriosis; the rate of preterm births in primiparous women with endometriosis was higher than in those without, while no differences were reported between multiparous women with or without endometriosis (124). In a Chinese retrospective study, a higher risk of preterm labor was found in 249 women with endometriosis compared with 249 controls who conceived naturally (125). The nationwide Swedish study conducted by Stephansson et al (126) found a higher risk of ART and adverse pregnancy outcomes, in particular preterm birth, in women with endometriosis compared to women without (126). Comparing women affected by endometriosis who conceived naturally or through ART, with fertile women with spontaneous pregnancies, Stern et al (127) retrospectively assessed an increased rate of preterm birth in the endometriosis non-ART group, but not in the endometriosis ART group. In their retrospective cohort study Fernando et al (128) underlined higher rates of preterm birth in infertile women with ovarian endometriomas compared to women with other endometriosis localizations.

\section{Small for gestational age}

In 2009, Fernando et al (128) found, for the first time, a link between ovarian endometriosis and the condition of small for gestational age (SGA). This retrospective cohort study distinguished women with different endometriosis localizations and infertile women both undergoing ART; they did not observe a higher risk of SGA in endometriosis in general, but they documented a statistically increased likelihood in women with ovarian endometriomas when compared with other forms of endometriosis. A large American retrospective study by Stern et al (127) demonstrated an association between SGA babies and women with endometriosis who conceived naturally, compared to affected women undergoing ART. Distinguishing for the first time primiparous and multiparous women with endometriosis, Conti et al (124) found a higher rate of SGA in both groups. However, some studies showed the opposite results. Stephansson et al (126) did not find a correlation between endometriosis and SGA babies. Benaglia et al (129) assessed a lower risk of SGA babies in women with ovarian endometriosis compared to other infertile women, both achieving pregnancy with ART techniques (129). The absence of a certain diagnosis of endometriosis, different control groups, different study designs, and the recruitment of women conceiving through ART - which itself 
is a risk factor for SGA babies - do not let us draw definitive conclusions on the association between the alteration of intrauterine growth and endometriosis.

\section{Hypertensive disorders and pre-eclampsia}

The elevated systemic inflammation found in women with endometriosis has been considered as a potential cause of pre-eclampsia and preterm birth. Although this association has been demonstrated in rodents, in human pregnancies the increased inflammatory status does not seem to cause the onset of hypertensive disorders and pre-eclampsia (130). Kortelahti et al (131) tried to evaluate, for the first time, the association between endometriosis and pre-eclampsia and found no correlation. However, the investigation shows some pitfalls: a small study population and a definition of pre-eclampsia not in line with the definition of the American College of Obstetricians and Gynecologists (ACOG) (131). Most studies published after the first report by Kortelahti et al (131) found no correlation between pregnancy hypertensive disorders and endometriosis (113-115, 124, 125, 127, 132, 133). A retrospective case-control study by Brosens et al (134) analyzed the incidence of pre-eclampsia and hypertensive disorders in infertile women with a clinical and/or laparoscopical diagnosis of endometriosis: the evaluation of the obstetrical outcomes showed a lower risk of pre-eclampsia in the endometriosis group. The large Swedish study by Stephansson et al (126) represented an exception; they observed an increased risk of pre-eclampsia among women with endometriosis. This study had some limitations: the analysis was stratified by ART only for the "preterm birth" outcome and no histological confirmation of the diagnosis of endometriosis after surgery was obtained. The controversial results of the literature do not allow us to draw firm conclusions on the association between endometriosis and hypertensive disorders of the pregnancy and the pre-eclampsia.

\section{Placenta previa}

The abnormal frequency and amplitude of uterine contractions, the impaired decidualization and placentation, and finally an anomalous blastocyst implantation could explain the higher incidence of placenta previa in women with endometriosis (135). The correlation between placenta previa and endometriosis has been demonstrated by a large number of studies. Saraswat et al (108), in their population-based cohort study comparing women with and without endometriosis, found a higher risk of adverse pregnancy outcomes beyond 24 weeks in the endometriosis group; in particular, they observed a higher incidence of placenta previa, in addition to unexplained antepartum/postpartum hemorrhage and cesarean section. A recent Chinese retrospective cohort study comparing 249 women with endometriosis and 249 women without endometriosis - all spontaneous singleton pregnancies - showed a higher risk of placenta previa in the subgroup of women affected by the disease (125). The Swedish nationwide cohort study by Stephansson et al (126) found a link between endometriosis and placental abnormalities, but did not specify the type of placental complication (126). Vercellini et al (113) studied the outcomes of spontaneous pregnancies in women undergoing surgery for endometriosis and stratified the results by the localization of endometriosis. Women with rectovaginal endometriosis had a sixfold increase in the risk of placenta previa compared to women with peritoneal and ovarian lesions. In a Japanese retrospective analysis of 318 pregnancies conceived by ART, endometriosis was strongly associated with placental complications, while age, parity, previous abortions, and ovulatory disorders were not related (136).

\section{Cesarean section}

Results regarding the higher likelihood of cesarean section in women with endometriosis are limited. The studies did not distinguish between natural or ART pregnancies, did not specify the principal indication of $C D$, and often did not divide prelabor and emergency CD. Conti et al (124) and Mekaru et al (115) found a similar rate of cesarean sections in women with endometriosis compared to controls. In contrast, a higher rate of cesarean sections was observed in women with ovarian and peritoneal endometriosis and in the group with rectovaginal lesions in the retrospective study conducted by Vercellini et al (113). Excluding pregnancies achieved by ART and adjusting for maternal age, Lin et al (125) showed an increased frequency of cesarean sections in the endometriosis group of a retrospective Chinese cohort study. Stephansson et al (126) found a higher risk of prelabor CD compared to emergency $C D$ in women with endometriosis without distinguishing spontaneous pregnancies from ART pregnancies. In a population-based study conducted by Glavind et al (137), women with endometriosis had an increased risk of cesarean section, and when stratified by the use of ART, the estimated risks remained essentially the same. We need well-designed studies to truly understand if endometriosis represents a risk factor for cesarean section.

\section{Postpartum hemorrhage}

The connection between endometriosis and postpartum hemorrhage has not been extensively analyzed. Most of the studies that try to explain this relationship did not distinguish between antepartum or postpartum bleeding and placental complications (126). An increased rate of uterine bleeding in infertile women with endometriosis was found in a retrospective study by Stern et al (127), although they did not specify the type of hemorrhage (ante or postpartum). Healy et al (138) studied the risk of postpartum hemorrhage in various groups of patients achieving pregnancy by ART and found an increased incidence in women with endometriosis. Glavind et al (137) found no association between endometriosis and postpartum hemorrhage in a population-based study using data from the Aarhus Birth Cohort, the largest European birth cohort. Drawing firm conclusions nowadays on the association between endometriosis and adverse pregnancy outcomes is difficult. The current evidence is based upon studies with many different designs and heterogeneous exposed and control groups. The authors often did not distinguish between spontaneous or ART pregnancies and did not stratify the results according to the age, body mass index, indication for modality of delivery, presence of adenomyosis 
and the stage and localizations of endometriosis. However, we can use these uncertain conclusions to counsel women with endometriosis and to consider their pregnancies more carefully.

\section{Conclusion}

Endometriosis is a benign gynecological disease that not only affects fertility (in terms of pregnancy rate) but also could negatively influence the pregnancy and neonatal outcomes (139). However, the risk of pregnancy and neonatal adverse complications is still debated. Greater knowledge of this disease should help avoid hasty obstetric decisions that could result in iatrogenic neonatal disease. Patients should also be fully informed about the potential for complications during their pregnancy. Adequate preconceptional counseling in women with endometriosis could be the best strategy for early detection of possible complications during pregnancy.

\section{Disclosures}

Financial support: No grants or funding have been received for this study.

Conflict of interest: None of the authors has financial interest related to this study to disclose.

\section{References}

1. Tanbo T, Fedorcsak P. Endometriosis-associated infertility: aspects of pathophysiological mechanisms and treatment options. Acta Obstet Gynecol Scand. 2017;96(6):659-667.

2. Harb HM, Gallos ID, Chu J, Harb M, Coomarasamy A. The effect of endometriosis on in vitro fertilisation outcome: a systematic review and meta-analysis. BJOG. 2013;120(11):1308-1320.

3. Lazzeri L, Di Giovanni A, Exacoustos C, et al. Preoperative and Postoperative Clinical and Transvaginal Ultrasound Findings of Adenomyosis in Patients With Deep Infiltrating Endometriosis. Reprod Sci. 2014;21(8):1027-1033.

4. DHooghe TM, Debrock S, Hill JA, Meuleman C. Endometriosis and subfertility: is the relationship resolved? Semin Reprod Med. 2003;21(2):243-254.

5. Opøien HK, Fedorcsak P, Omland AK, et al. In vitro fertilization is a successful treatment in endometriosis-associated infertility. Fertil Steril. 2012;97(4):912-918.

6. Dunselman GA, Vermeulen N, Becker C, et al. European Society of Human Reproduction and Embryology. ESHRE guideline: management of women with endometriosis. Hum Reprod. 2014;29(3):400-412.

7. Roman H. Colorectal endometriosis and pregnancy wish: why doing primary surgery. Front Biosci (Schol Ed). 2015;7(7):83-93.

8. Leone Roberti Maggiore U, Ferrero S, Mangili G, et al. A systematic review on endometriosis during pregnancy: diagnosis, misdiagnosis, complications and outcomes. Hum Reprod Update. 2015;0(0):1-34.

9. Jabbour HN, Sales KJ, Catalano RD, Norman JE. Inflammatory pathways in female reproductive health and disease. Reproduction. 2009;138(6):903-919.

10. Manresa MC, Godson C, Taylor CT. Hypoxia-sensitive pathways in inflammation-driven fibrosis. Am J Physiol Regul Integr Comp Physiol. 2014;307(12):R1369-R1380.

11. OLeary SM. Ectopic decidualization causing massive postpartum intraperitoneal hemorrhage. Obstet Gynecol. 2006;108 (3 Pt 2):776-779.
12. Erikson DW, Chen JC, Piltonen TT, Conti M, Irwin JC, Giudice LC. Inhibition of epidermal growth factor receptor restores decidualization markers in stro- mal fibroblasts from women with endometriosis. J Endometriosis. 2014;6:196-211.

13. Redwine DB. Ovarian endometriosis: a marker for more extensive pelvic and intestinal disease. Fertil Steril. 1999;72(2):310-315.

14. Guerriero $S$, Ajossa $S$, Orozco R, et al. Accuracy of transvaginal ultrasound for diagnosis of deep endometriosis in the rectosigmoid: systematic review and meta-analysis. Ultrasound Obstet Gynecol. 2016;47(3):281-289.

15. Senapati S, Sammel MD, Morse C, Barnhart KT. Impact of endometriosis on in vitro fertilization outcomes: an evaluation of the Society for Assisted Reproductive Technologies Database. Fertil Steril. 2016;106(1):164-171.e1.

16. Vercellini $P$, Viganò $P$, Somigliana E, Fedele L. Endometriosis: pathogenesis and treatment. Nat Rev Endocrinol. 2014;10(5):261-275.

17. Sanchez AM, Vanni VS, Bartiromo L, et al. Is the oocyte quality affected by endometriosis? A review of the literature. J Ovarian Res. 2017;10(1):43.

18. Maneschi F, Marasá L, Incandela S, Mazzarese M, Zupi E. Ovarian cortex surrounding benign neoplasms: a histologic study. Am J Obstet Gynecol. 1993;169(2 Pt 1):388-393.

19. Benaglia L, Somigliana E, Vercellini P, Abbiati A, Ragni G, Fedele L. Endometriotic ovarian cysts negatively affect the rate of spontaneous ovulation. Hum Reprod. 2009;24(9): 2183-2186

20. Goodman LR, Goldberg JM, Flyckt RL, Gupta M, Harwalker J, Falcone T. Effect of surgery on ovarian reserve in women with endometriomas, endometriosis and controls. Am J Obstet Gynecol. 2016;215(5):589.e1-589.e6.

21. Uncu G, Kasapoglu I, Ozerkan K, Seyhan A, Oral Yilmaztepe A, Ata B. Prospective assessment of the impact of endometriomas and their removal on ovarian reserve and determinants of the rate of decline in ovarian reserve. Hum Reprod. 2013;28(8):2140-2145.

22. Chen $\mathrm{Y}$, Pei $\mathrm{H}$, Chang $\mathrm{Y}$, et al. The impact of endometrioma and laparoscopic cystectomy on ovarian reserve and the exploration of related factors assessed by serum anti-Mullerian hormone: a prospective cohort study. J Ovarian Res. 2014;7:108.

23. Raffi F, Metwally M, Amer S. The impact of excision of ovarian endometrioma on ovarian reserve: a systematic review and meta-analysis. J Clin Endocrinol Metab. 2012;97(9):3146-3154.

24. Somigliana E, Berlanda N, Benaglia L, Viganò P, Vercellini $P$, Fedele L. Surgical excision of endometriomas and ovarian reserve: a systematic review on serum antimüllerian hormone level modifications. Fertil Steril. 2012;98(6):1531-1538.

25. Goodman LR, Goldberg JM, Flyckt RL, et al. Effect of surgery on ovarian reserve in women with endometriomas, endometriosis, and controls. Am J Obstet Gynecol. 2016;215(5): 589e1-589e6.

26. Vignali M, Mabrouk M, Ciocca E, et al. Surgical excision of ovarian endometriomas: Does it truly impair ovarian reserve? Long term anti-Müllerian hormone (AMH) changes after surgery. J Obstet Gynaecol Res. 2015;41(11):1773-1778.

27. Sugita A, Iwase A, Goto $M$, et al. One-year follow-up of serum antimüllerian hormone levels in patients with cystectomy: are different sequential changes due to different mechanisms causing damage to the ovarian reserve? Fertil Steril. 2013;100(2):516-22.e3.

28. Singh SS, Suen MW. Surgery for endometriosis: beyond medical therapies. Fertil Steril. 2017;107(3):549-554.

29. Kennedy S, Bergqvist A, Chapron C, et al. ESHRE Special Interest Group for Endometriosis and Endometrium Guideline Development Group. ESHRE guideline for the diagnosis and treatment of endometriosis. Hum Reprod. 2005;20(10):2698-2704. 
30. Chapron $C$, Vercellini $P$, Barakat $H$, Vieira $M$, Dubuisson JB. Management of ovarian endometriomas. Hum Reprod Update. 2002;8(6):591-597.

31. Vercellini P, Chapron C, De Giorgi O, Consonni D, Frontino G, Crosignani PG. Coagulation or excision of ovarian endometriomas? Am J Obstet Gynecol. 2003;188(3):606-610.

32. Beretta P, Franchi M, Ghezzi F, Busacca M, Zupi E, Bolis P. Randomized clinical trial of two laparoscopic treatments of endometriomas: cystectomy versus drainage and coagulation. Fertil Steril. 1998;70(6):1176-1180.

33. Somigliana $E$, Arnoldi $M$, Benaglia $L$, lemmello $R$, Nicolosi $A E$, Ragni G. IVF-ICSI outcome in women operated on for bilateral endometriomas. Hum Reprod. 2008;23(7):1526-1530.

34. Somigliana E, Infantino M, Benedetti F, Arnoldi M, Calanna G, Ragni $G$. The presence of ovarian endometriomas is associated with a reduced responsiveness to gonadotropins. Fertil Steril. 2006;86(1):192-196.

35. Sanchez AM, Viganò $P$, Somigliana $E$, Panina-Bordignon $P$, Vercellini $\mathrm{P}$, Candiani $\mathrm{M}$. The distinguishing cellular and molecular features of the endometriotic ovarian cyst: from pathophysiology to the potential endometrioma-mediated damage to the ovary. Hum Reprod Update. 2014;20(2):217-230.

36. Sanchez AM, Vanni VS, Bartiromo L, et al. Is the oocyte quality affected by endometriosis? A review of the literature. J Ovarian Res. 2017;10(1):43.

37. Rossi AC, Prefumo F. The effects of surgery for endometriosis on pregnancy outcomes following in vitro fertilization and embryo transfer: a systematic review and meta-analysis. Arch Gynecol Obstet. 2016;294(3):647-655.

38. Duffy JM, Arambage K, Correa FJ, et al. Laparoscopic surgery for endometriosis. Cochrane Database Syst Rev. 2014;(4):CD011031.

39. Hamdan M, Dunselman G, Li TC, Cheong Y. The impact of endometrioma on IVF/ICSI outcomes: a systematic review and metaanalysis. Hum Reprod Update. 2015;21(6):809-825.

40. Alborzi S, Keramati P, Younesi M, Samsami A, Dadras N. The impact of laparoscopic cystectomy on ovarian reserve in patients with unilateral and bilateral endometriomas. Fertil Steril. 2014;101(2):427-434.

41. Muzii L, Achilli $C$, Lecce $F$, et al. Second surgery for recurrent endometriomas is more harmful to healthy ovarian tissue and ovarian reserve than first surgery. Fertil Steril. 2015;103(3): 738-743.

42. Streuli I, de Ziegler D, Gayet V, et al. In women with endometriosis anti-Müllerian hormone levels are decreased only in those with previous endometrioma surgery. Hum Reprod. 2012;27(11):3294-3303.

43. Chapron C, Pietin-Vialle C, Borghese B, Davy C, Foulot H, Chopin N. Associated ovarian endometrioma is a marker for greater severity of deeply infiltrating endometriosis. Fertil Steril. 2009;92(2):453-457.

44. Martinez A, Howard FM. The efficacy of laparoscopic surgical treatment of ovarian remnant and ovarian retention syndromes. J Minim Invasive Gynecol. 2015;22(2):245-249.

45. Shervin A, Mohazzab A, Aminlou M, et al. Fertility outcome after laparoscopic treatment of advanced endometriosis in two groups of infertile patients with and without ovarian endometrioma. Eur J Obstet Gynecol Reprod Biol. 2016;201:46-50.

46. Cranney R, Condous G, Reid S. An update on the diagnosis, surgical management, and fertility outcomes for women with endometrioma. Acta Obstet Gynecol Scand. 2017;96(6): 633-643.

47. Balasch J, Creus M, Fábregues F, et al. Visible and non-visible endometriosis at laparoscopy in fertile and infertile women and in patients with chronic pelvic pain: a prospective study. Hum Reprod. 1996;11(2):387-391.
48. Santulli P, Lamau MC, Marcellin L, et al. Endometriosis-related infertility: ovarian endometrioma per se is not associated with presentation for infertility. Hum Reprod. 2016;31(8): 1765-1775.

49. Marcoux S, Maheux R, Bérubé S, Canadian Collaborative Group on Endometriosis. Laparoscopic surgery in infertile women with minimal or mild endometriosis. N Engl J Med. 1997;337(4):217-222.

50. Vercellini $P$, Crosignani PG. Minimal and mild endometriosis. Is there anything new under the sun? J Reprod Med. 1993;38(1):49-52.

51. Younis JS, Laufer N. Peritoneal fluid in the pouch of Douglas: strategically located and affecting reproductive events. Fertil Steril. 2015;104(4):831-832.

52. Jacobson TZ, Duffy JM, Barlow D, Farquhar C, Koninckx PR, Olive D. Laparoscopic surgery for subfertility associated with endometriosis. Cochrane Database Syst Rev. 2010;(1):CD001398.

53. Brichant G, Audebert A, Nisolle M. Minimal and mild endometriosis: which impact on fertility? Rev Med Liege. 2016;71(5): 236-241.

54. Boujenah J, Cedrin-Durnerin I, Herbemont C, Sifer C, Poncelet C. Non-ART pregnancy predictive factors in infertile patients with peritoneal superficial endometriosis. Eur J Obstet Gynecol Reprod Biol. 2017;211:182-187.

55. Opøien HK, Fedorcsak P, Byholm T, Tanbo T. Complete surgical removal of minimal and mild endometriosis improves outcome of subsequent IVF/ICSI treatment. Reprod Biomed Online. 2011;23(3):389-395.

56. van der Houwen LE, Schreurs AM, Schats R, et al. Efficacy and safety of intrauterine insemination in patients with moderate-to-severe endometriosis. Reprod Biomed Online. 2014;28(5):590-598.

57. Werbrouck E, Spiessens C, Meuleman C, DHooghe T. No difference in cycle pregnancy rate and in cumulative live-birth rate between women with surgically treated minimal to mild endometriosis and women with unexplained infertility after controlled ovarian hyperstimulation and intrauterine insemination. Fertil Steril. 2006;86(3):566-571.

58. Tummon IS, Asher LJ, Martin JS, Tulandi T. Randomized controlled trial of superovulation and insemination for infertility associated with minimal or mild endometriosis. Fertil Steril. 1997;68(1):8-12.

59. Guzick DS, Carson SA, Coutifaris C, et al. National Cooperative Reproductive Medicine Network. Efficacy of superovulation and intrauterine insemination in the treatment of infertility. $\mathrm{N}$ Engl J Med. 1999;340(3):177-183.

60. Fedele L, Bianchi S, Marchini M, Villa L, Brioschi D, Parazzini F. Superovulation with human menopausal gonadotropins in the treatment of infertility associated with minimal or mild endometriosis: a controlled randomized study. Fertil Steril. 1992;58(1):28-31.

61. Gandhi AR, Carvalho LF, Nutter B, Falcone T. Determining the fertility benefit of controlled ovarian hyperstimulation with intrauterine insemination after operative laparoscopy in patients with endometriosis. J Minim Invasive Gynecol. 2014;21(1):101-108.

62. Parazzini F. Ablation of lesions or no treatment in minimalmild endometriosis in infertile women: a randomized trial. Gruppo Italiano per lo Studio dellEndometriosi. Hum Reprod. 1999;14(5):1332-1334.

63. Maccagnano C, Pellucchi F, Rocchini L, et al. Ureteral endometriosis: proposal for a diagnostic and therapeutic algorithm with a review of the literature. Urol Int. 2013;91 (1):1-9.

64. Knabben L, Imboden S, Fellmann B, Nirgianakis K, Kuhn A, Mueller MD. Urinary tract endometriosis in patients with deep infiltratingendometriosis:prevalence, symptoms, management, 
and proposal for a new clinical classification. Fertil Steril. 2015; 103(1):147-152.

65. Maccagnano $C$, Pellucchi $F$, Rocchini L, et al. Diagnosis and treatment of bladder endometriosis: state of the art. Urol Int. 2012;89(3):249-258.

66. Shook TE, Nyberg LM. Endometriosis of the urinary tract. Urology. 1988;31(1):1-6.

67. Seracchioli R, Mabrouk M, Montanari G, Manuzzi L, Concetti S, Venturoli S. Conservative laparoscopic management of urinary tract endometriosis (UTE): surgical outcome and long-term follow-up. Fertil Steril. 2010;94(3):856-861.

68. Donnez J, Nisolle M, Squifflet J. Ureteral endometriosis: a complication of rectovaginal endometriotic (adenomyotic) nodules. Fertil Steril. 2002;77(1):32-37.

69. Kjer JJ, Kristensen J, Hartwell D, Jensen MA. Full-thickness endometriosis of the bladder: report of 31 cases. Eur J Obstet Gynecol Reprod Biol. 2014;176:31-33.

70. Saavalainen L, Heikinheimo O, Tiitinen A, Härkki P. Deep infiltrating endometriosis affecting the urinary tract-surgical treatment and fertility outcomes in 20042013. Gynecol Surg. 2016;13(4):435-444.

71. Soriano D, Bouaziz J, Elizur S, et al. Reproductive outcome is favorable after laparoscopic resection of bladder endometriosis. J Minim Invasive Gynecol. 2016;23(5):781-786.

72. Daraï E, Lesieur B, Dubernard G, Rouzier R, Bazot M, Ballester M. Fertility after colorectal resection for endometriosis: results of a prospective study comparing laparoscopy with open surgery. Fertil Steril. 2011;95(6):1903-1908.

73. Tarjanne S, Heikinheimo O, Mentula M, Härkki P. Complications and long-term follow-up on colorectal resections in the treatment of deep infiltrating endometriosis extending to bowel wall. Acta Obstet Gynecol Scand. 2015;94(1):72-79.

74. Vercellini P, Parazzini F, Pietropaolo G, Cipriani S, Frattaruolo MP, Fedele L. Pregnancy outcome in women with peritoneal, ovarian and rectovaginal endometriosis: a retrospective cohort study. BJOG. 2012;119(12):1538-1543.

75. Fadhlaoui A, Bouquet de la Jolinière J, Feki A. Endometriosis and infertility: how and when to treat? Front Surg. 2014; 1:24.

76. Brown J, Farquhar C. Endometriosis: an overview of Cochrane Reviews. Cochrane Database Syst Rev. 2014;(3):CD009590.

77. Coronado C, Franklin RR, Lotze EC, Bailey HR, Valdés CT. Surgical treatment of symptomatic colorectal endometriosis. Fertil Steril. 1990;53(3):411-416.

78. Duepree HJ, Senagore AJ, Delaney CP, Marcello PW, Brady KM, Falcone T. Laparoscopic resection of deep pelvic endometriosis with rectosigmoid involvement. J Am Coll Surg. 2002; 195(6):754-758.

79. Abbott JA, Hawe J, Clayton RD, Garry R. The effects and effectiveness of laparoscopic excision of endometriosis: a prospective study with 25 year follow-up. Hum Reprod. 2003;18(9): 1922-1927.

80. Kavallaris A, Köhler C, Kühne-Heid R, Schneider A. Histopathological extent of rectal invasion by rectovaginal endometriosis. Hum Reprod. 2003;18(6):1323-1327.

81. Ford J, English J, Miles WA, Giannopoulos T. Pain, quality of life and complications following the radical resection of rectovaginal endometriosis. BJOG. 2004;111(4):353-356.

82. Fleisch MC, Xafis D, De Bruyne F, Hucke J, Bender HG, Dall P. Radical resection of invasive endometriosis with bowel or bladder involvementlong-term results. Eur J Obstet Gynecol Reprod Biol. 2005;123(2):224-229.

83. Dubernard G, Piketty M, Rouzier R, Houry S, Bazot M, Darai E. Quality of life after laparoscopic colorectal resection for endometriosis. Hum Reprod. 2006;21(5):1243-1247.
84. Landi S, Ceccaroni M, Perutelli A, et al. Laparoscopic nervesparing complete excision of deep endometriosis: is it feasible? Hum Reprod. 2006;21(3):774-781.

85. Alabiso G, Alio L, Arena S, et al. Endometriosis Treatment Italian Club. Adenomyosis: What the Patient Needs. J Minim Invasive Gynecol. 2016;23(4):476-488.

86. Adamson GD, Pasta DJ. Endometriosis fertility index: the new, validated endometriosis staging system. Fertil Steril. 2010;94(5):1609-1615.

87. Stepniewska A, Pomini P, Bruni F, et al. Laparoscopic treatment of bowel endometriosis in infertile women. Hum Reprod. 2009;24(7):1619-1625.

88. Daraï E, Cohen J, Ballester M. Colorectal endometriosis and fertility. Eur J Obstet Gynecol Reprod Biol. 2017;209:86-94.

89. Centini G, Afors K, Murtada R, et al. Impact of Laparoscopic Surgical Management of Deep Endometriosis on Pregnancy Rate. J Minim Invasive Gynecol. 2016;23(1):113-119.

90. Vigano P, Corti L, Berlanda N. Beyond infertility: obstetrical and postpartum complications associated with endometriosis and adenomyosis. Fertil Steril. 2015;104(4):802-812.

91. Brosens IA, Fusi L, Brosens JJ. Endometriosis is a risk factor for spontaneous hemoperitoneum during pregnancy. Fertil Steril. 2009;92(4):1243-1245.

92. Plaisier M. Decidualisation and angiogenesis. Best Pract Res Clin Obstet Gynaecol. 2011;25(3):259-271.

93. Gao JL, Lortie K, Singh SS. Laparoscopic internal iliac artery ligation for postpartum spontaneous hemoperitoneum. J Obstet Gynaecol Can. 2010;32(12):1172-1175.

94. Remorgida V, Ferrero S, Fulcheri E, Ragni N, Martin DC. Bowel endometriosis: presentation, diagnosis, and treatment. Obstet Gynecol Surv. 2007;62(7):461-470.

95. Setúbal A, Sidiropoulou Z, Torgal M, Casal E, Lourenço C, Koninckx P. Bowel complications of deep endometriosis during pregnancy or in vitro fertilization. Fertil Steril. 2014;101(2): 442-446.

96. Van De Putte I, Campo R, Gordts S, Brosens I. Uterine rupture following laparoscopic resection of rectovaginal endometriosis: a new risk factor? Br J Obstet Gynaecol. 1999;106(6): 608-609.

97. Granese R. Acute abdomen in pregnancy with endometriosis. Acta Obstet Gynecol Scand. 2010;89(6):844-845.

98. Chen ZHY, Chen M, Tsai H-D, Wu C-H. Intrapartum uterine rupture associated with a scarred cervix because of a previous rupture of cystic cervical endometriosis. Taiwan J Obstet Gynecol. 2011;50(1):95-97.

99. Sholapurkar SL, Sharp NC, Hirschowitz L. Life-threatening uterine haemorrhage six weeks after Caesarean section due to uterine scar endometriosis: case report and review of literature. Aust N Z J Obstet Gynaecol. 2005;45(3):256-258.

100. Zwart JJ, Richters JM, Ory F, de Vries JI, Bloemenkamp KW, van Roosmalen J. Uterine rupture in The Netherlands: a nationwide population-based cohort study. BJOG. 2009;116(8):10691078, discussion 1078-1080.

101. Chester JM, Israfil-Bayli F. Endometriosis: a possible cause of uterine rupture in labour? J Obstet Gynaecol. 2015;35(6):638.

102. Petraglia F, Arcuri F, de Ziegler D, Chapron C. Inflammation: a link between endometriosis and preterm birth. Fertil Steril. 2012;98(1):36-40.

103. Brosens I, Brosens JJ, Fusi L, Al-Sabbagh M, Kuroda K, Benagiano G. Risks of adverse pregnancy outcome in endometriosis. Fertil Steril. 2012a;98(1):30-35.

104. Viganò $P$, Somigliana E, Panina $P$, Rabellotti E, Vercellini $P$, Candiani M. Principles of phenomics in endometriosis. Hum Reprod Update. 2012;18(3):248-259.

105. Groll M. Endometriosis and spontaneous abortion. Fertil Steril. 1984;41(6):933-935. 
106. Naples JD, Batt RE, Sadigh H. Spontaneous abortion rate in patients with endometriosis. Obstet Gynecol. 1981;57(4): 509-512.

107. Wheeler JM, Johnston BM, Malinak LR. The relationship of endometriosis to spontaneous abortion. Fertil Steril. 1983; 39(5):656-660.

108. Saraswat L, Ayansina DT, Cooper KG, et al. Pregnancy outcome in women with endometriosis: a national record linkage study. BJOG. 2017; 124(3):444-452.

109. FitzSimmons J, Stahl R, Gocial B, Shapiro SS. Spontaneous abortion and endometriosis. Fertil Steril. 1987;47(4):696-698.

110. Candiani GB, Danesino V, Gastaldi A, Parazzini F, Ferraroni M. Reproductive and menstrual factors and risk of peritoneal and ovarian endometriosis. Fertil Steril. 1991;56(2):230-234.

111. Omland AK, Abyholm T, Fedorcsák P, et al. Pregnancy outcome after IVF and ICSI in unexplained, endometriosis-associated and tubal factor infertility. Hum Reprod. 2005;20(3): 722-727.

112. Gergolet M, Gianaroli L, Kenda Suster N, Verdenik I, Magli MC, Gordts S. Possible role of endometriosis in the aetiology of spontaneous miscarriage in patients with septate uterus. Reprod Biomed Online. 2010;21(4):581-585.

113. Vercellini P, Parazzini F, Pietropaolo G, Cipriani S, Frattaruolo MP, Fedele L. Pregnancy outcome in women with peritoneal, ovarian and rectovaginal endometriosis: a retrospective cohort study. BJOG. 2012;119(12):1538-1543.

114. Aris A. A 12-year cohort study on adverse pregnancy outcomes in Eastern Townships of Canada: impact of endometriosis. Gynecol Endocrinol. 2014;30(1):34-37.

115. Mekaru K, Masamoto $H$, Sugiyama $H$, et al. Endometriosis and pregnancy outcome: are pregnancies complicated by endometriosis a high-risk group? Eur J Obstet Gynecol Reprod Biol. 2014;172:36-39.

116. Matorras R, Rodríguez F, Gutierrez de Terán G, Pijoan JI, Ramón $\mathrm{O}$, Rodríguez-Escudero FJ. Endometriosis and spontaneous abortion rate: a cohort study in infertile women. Eur J Obstet Gynecol Reprod Biol. 1998;77(1):101-105.

117. Hjordt Hansen MV, Dalsgaard T, Hartwell D, Skovlund CW, Lidegaard O. Reproductive prognosis in endometriosis. A national cohort study. Acta Obstet Gynecol Scand. 2014;93(5): 483-489.

118. Barbosa MA, Teixeira DM, Navarro PA, Ferriani RA, Nastri CO, Martins WP. Impact of endometriosis and its staging on assisted reproduction outcome: systematic review and metaanalysis. Ultrasound Obstet Gynecol. 2014;44(3):261-278.

119. Leonardi $M$, Papaleo $E$, Reschini $M$, et al. Risk of miscarriage in women with endometriosis: insights from in vitro fertilization cycles. Fertil Steril. 2016;106(2):386-392.e3.

120. Pittaway DE, Vernon C, Fayez JA. Spontaneous abortions in women with endometriosis. Fertil Steril. 1988;50(5): 711-715.

121. Marcoux S, Maheux R, Bérubé S; Canadian Collaborative Group on Endometriosis. Laparoscopic surgery in infertile women with minimal or mild endometriosis. N Engl J Med. 1997;337(4):217-222.

122. Gruppo Italiano per lo Studio dell'Endometriosi. Ablation of lesions or no treatment in minimal-mild endometriosis in infertile women: a randomized trial. Hum Reprod. 1999;14:1332-1334.
123. Schieve LA, Ferre C, Peterson HB, Macaluso M, Reynolds MA Wright VC. Perinatal outcome among singleton infants conceived through assisted reproductive technology in the United States. Obstet Gynecol. 2004;103(6):1144-1153.

124. Conti N, Cevenini G, Vannuccini S, et al. Women with endometriosis at first pregnancy have an increased risk of adverse obstetric outcome. J Matern Fetal Neonatal Med. 2014;9:1-4.

125. Lin H, Leng JH, Liu JT, Lang JH. Obstetric outcomes in Chinese women with endometriosis: a retrospective cohort study. Chin Med J (Engl). 2015;128(4):455-458.

126. Stephansson O, Kieler H, Granath F, Falconer H. Endometriosis, assisted reproduction technology, and risk of adverse pregnancy outcome. Hum Reprod. 2009;24(9):2341-2347.

127. Stern JE, Luke B, Tobias M, Gopal D, Hornstein MD, Diop H. Adverse pregnancy and birth outcomes associated with underlying diagnosis with and without assisted reproductive technology treatment. Fertil Steril. 2015;103(6):1438-1445.

128. Fernando S, Breheny S, Jaques AM, Halliday JL, Baker G, Healy D. Preterm birth, ovarian endometriomata, and assisted reproduction technologies. Fertil Steril. 2009;91(2):325-330.

129. Benaglia L, Bermejo A, Somigliana E, et al. Pregnancy outcome in women with endometriomas achieving pregnancy through IVF. Hum Reprod. 2012;27(6):1663-1667.

130. Ahmed A, Ramma W. Unravelling the theories of pre-eclampsia: are the protective pathways the new paradigm? Br J Pharmacol. 2015;172(6):1574-1586.

131. Kortelahti M, Anttila MA. Hippeläinen MI, Heinonen ST. Obstetric outcome in women with endometriosis-a matched case control study. Gynecol Obstet Invest. 2003;56:207-212.

132. Hadfield RM, Lain SJ, Raynes-Greenow CH, Morris JM, Roberts $\mathrm{CL}$. Is there an association between endometriosis and the risk of pre-eclampsia? A population based study. Hum Reprod. 2009;24(9):2348-2352.

133. Kuivasaari-Pirinen $P$, Raatikainen $K$, Hippeläinen $M$, Heinonen S. Adverse outcomes of IVF/ICSI pregnancies vary depending on aetiology of infertility. ISRN Obstet Gynecol. 2012;2012: Article ID:451915.

134. Brosens IA, De Sutter P, Hamerlynck T, et al. Endometriosis is associated with a decreased risk of pre-eclampsia. Hum Reprod. 2007;22(6):1725-1729.

135. Kunz G, Beil D, Huppert P, Leyendecker G. Structural abnormalities of the uterine wall in women with endometriosis and infertility visualized by vaginal sonography and magnetic resonance imaging. Hum Reprod. 2000;15(1):76-82.

136. Takemura $Y$, Osuga $Y$, Fujimoto $A$, et al. Increased risk of placenta previa is associated with endometriosis and tubal factor infertility in assisted reproductive technology pregnancy. Gynecol Endocrinol. 2013;29(2):113-115.

137. Glavind MT, Forman A, Arendt LH, Nielsen K, Henriksen TB. Endometriosis and pregnancy complications: a Danish cohort study. Fertil Steril. 2017;107(1):160-166.

138. Healy DL, Breheny S, Halliday J, et al. Prevalence and risk factors for obstetric haemorrhage in 6730 singleton births after assisted reproductive technology in Victoria Australia. Hum Reprod. 2010;25(1):265-274.

139. Exacoustos C, Lauriola I, Lazzeri L, De Felice G, Zupi E. Complications during pregnancy and delivery in women with untreated rectovaginal deep infiltrating endometriosis. Fertil Steril. 2016;106(5):1129-1135.e1. 